\title{
Hypersensitivity and cross-reactivity to cisplatin and carboplatin
}

\author{
Gholamreza Faridaalaee', Seyed Hesam Rahmani2 ${ }^{2}$, Amin Mahboubi ${ }^{1}$ \\ 'Emergency Medicine Department, Maragheh University of Medical Sciences, Maragheh, Iran \\ 2Emergency Medicine Department, Urmia University of Medical Sciences, Urmia, Iran
}

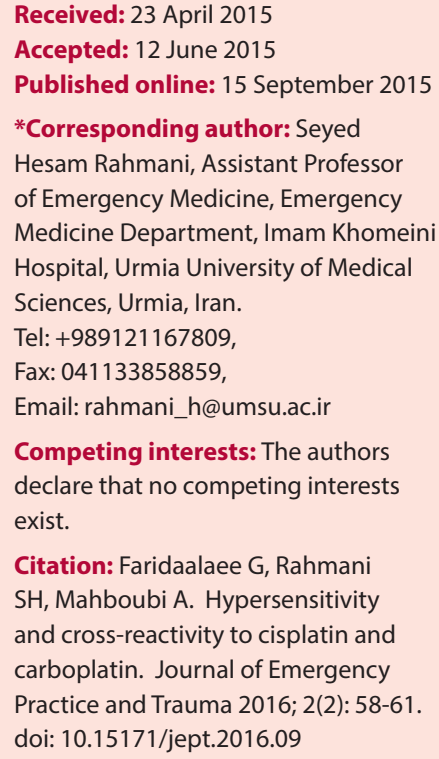

\begin{abstract}
Cisplatin was the first of the platinum drugs. Second-generation platinum derivative was carboplatin that its efficacy in the treatment of many malignancies is equal to cisplatin, and its toxicity profile is more favorable. Here we report on a 50-year-old woman with a history of cervix cancer who developed a severe hypersensitivity reaction (HSR) to carboplatin. She was admitted to the emergency department (ED) with shortness of breath, tachypnea, restless, agitation, and lethargy. On arrival, the patient was hemodynamically unstable; we initiated treatment immediately with hydration, oxygen therapy with mask, hydrocortisone, midazolam, and adrenalin. After 1 hour, BP and $\mathrm{O}_{2}$ sat improved to $100 / 70 \mathrm{~mm} \mathrm{Hg}$ and $92 \%$ respectively, but there was not any significant improvement in tachycardia as well as tachypnea and she was still lethargic and agitated. Her symptoms improved gradually after 18 hours of admission. She was discharged after 36 hours. HSRs to cisplatin and carboplatin can be potentially lifethreatening. The symptoms can range from a mild rash to severe anaphylaxis. Doctors should be aware of these reactions, determine appropriate treatment, and know the cross-reactivity among these drugs.
\end{abstract}

Keywords: Cisplatin, Carboplatin, Cross-reactivity, Cancer, Anaphylaxis

\section{Introduction}

Platinum-based compounds were first synthesized in the nineteenth century and their clinical use against cancer started in the 1970s $(1,2)$. These drugs have been approved for the treatment of numerous malignancies, such as ovarian, primary peritoneal carcinoma, bladder, head and neck, colorectal, pancreatic, esophageal, gastric, testicular, endometrial, biliary tract, lung cancer, and mesothelioma (3). Cisplatin was the first of the platinum drugs to be used (1). Second-generation platinum derivative carboplatin differs from cisplatin in the substitution of 2 chlorides. Its efficacy in the treatment of many of the above malignancies is equal to that of cisplatin, and its toxicity profile is more favorable. Therefore, carboplatin has often been used in place of cisplatin $(4,5)$. Cisplatin has some toxicity and adverse effects such as nausea, vomiting, anorexia, diarrhea, constipation, tinnitus, taste alteration, alopecia, hypocalcaemia, nephrotoxicity, and peripheral neurotoxicity $(6,7)$. Several side effects of cisplatin such as hypersensitivity and electrolyte disturbance are uncommon $(8,9)$. Hypersensitivity reactions (HSRs) in patients receiving cisplatin was first described in the 1970s in patients who had been retreated with this drug (10). Extensive use of platinum compounds in chemotherapy during the last decade has led to a significant increase in the incidence of HSRs. Hypersensitivity to carboplatin is rarely observed during the first course of treatment $(11,12)$. During the first five cycles, the overall risk is less than $1 \%$ and it rises sharply to $6.5 \%$ with the sixth cycle and has been reported as high as $27 \%$ in patients receiving more than seven cycles of treatment $(13,14)$. The incidence of cisplatin hypersensitivity exhibits similar characteristics to those observed with carboplatin. It seems to range from $5 \%$ to $20 \%$ and increases with concomitant radiation (11).

\section{Case report}

A 50-year-old female was admitted to the emergency department (ED) with shortness of breath, tachypnea, restless, agitation, and lethargy. She had a history of cervix cancer during the past 2 months and had undergone total hysterectomy and chemotherapy. She was treated with carboplatin and taxol for the first cycle. Unfortunately, 
she experienced severe HSR during infusion of carboplatin. This reaction occurred during one fifth of the way through a 700-mg carboplatin infusion. The palpitation and short of breath was controlled with $200 \mathrm{mg}$ intravenous hydrocortisone. Therefore, in the second cycle, carboplatin changed to cisplatin, and hydrocortisone 100 mg IV, cimetidine $200 \mathrm{mg}$ IV and chlorpheniramine IM were injected before administration. However, she experienced severe HSR again with hypotension, palpitation, and shortness of breath during administration of cisplatin. This reaction occurred during one half of the way through a cisplatin. She referred to us immediately. On arrival, the patient was hemodynamically unstable. Oral temperature was $36.3^{\circ} \mathrm{C}$, pulse rate 152 beats per minute with a normal rhythm, blood pressure $90 / 60 \mathrm{~mm} \mathrm{Hg}$, and his respiratory rate was 26 breaths per minute with no abnormal breath sounds. The initial oxygen saturation was $75 \%$ (in room air). She was confused and somnolent with apparent respiratory distress. A 12-lead electrocardiogram revealed sinus tachycardia at a rate of $152 \mathrm{bpm}$, a complete blood count was normal except for a mildly elevated white blood cell count of 11.6-103/mL (11.6-109/L), hemoglobin 11.2 $\mathrm{g} / \mathrm{dL}$. His serum blood urea nitrogen, creatinine, creatinine kinase, and liver function tests were normal. Coagulation studies, including a prothrombin time and activated partial thromboplastin time, were normal. Serum glucose was $110 \mathrm{mg} / \mathrm{dL}$. There was respiratory alkalosis in arterial blood gases analysis. We initiated treatment immediately with hydration (normal saline $1 \mathrm{~L}$ in 1 hour ), O2 $7 \mathrm{~L} /$ min with mask, hydrocortisone $200 \mathrm{mg}$ and midazolam 2 mg IV slowly and adrenalin $0.4 \mathrm{mg}$ (1:1000) IM. After 1 hour, BP and O2 sat improved to 100/70 mm Hg and 92\% respectively, but there was not any significant improvement in sinus tachycardia as well as tachypnea and she was still lethargic and agitated. After 3 hours with regard to any improvement in the level of consciousness, brain computed tomography (CT) scan was performed and due to doubtful hyperdense lesion, brain magnetic resonance imaging (MRI) was performed too in which it was normal. Her symptoms improved gradually after 18 hours of admission and became normal after 24 hours. The patient was observed for 36 hours and discharged after oncology consultation for outpatient follow-up.

\section{Discussion}

HSRs have been reported to carboplatin too. Carboplatin has a black box warning: "Anaphylactic-like reactions may occur within minutes of administration. Epinephrine, corticosteroids, and antihistamines may alleviate symptoms." Several reports have indicated switching to cisplatin without the complications of a HSR (15). Cisplatin also has a black box warning: "Anaphylactic-like reactions have occurred. Facial edema, bronchoconstriction, tachycardia, and hypotension may occur within minutes of cisplatin administration. Epinephrine, corticosteroids, and antihistamines have been effectively employed to alleviate symptoms (16). Although hypersensitivity to cisplatin has been reported to occur in $1 \%$ to $20 \%$ of patients, carboplatin has rarely been implicated as a cause of HSRs $(17,18)$. Platinum hypersensitivity symptoms may develop acutely during infusion or within minutes, hours, or days after the infusion, in patients receiving carboplatin, the number of prior platinum treatments and total lifetime exposure is associated with the possibility of hypersensitivity (12). We believe there was a hypersensitivity and cross-reactivity to both carboplatin and cisplatin in this case, although some studies have reported safely administration of cisplatin in patients with a history of carboplatin-allergic reactions (19-21). Successful replacement of carboplatin by cisplatin has been demonstrated in women with gynecological malignancies but the true incidence of cross-reactivity between platinum salts is not yet known. The possibility of developing a reaction to the platinum group agent may be as high as $25 \%$ and cases of fatal cisplatin reactions after carboplatin hypersensitivity have been reported $(20,22)$. Kook et al (13) in 1998 reported on an 8-year-old girl with life-threatening carboplatin hypersensitivity during conditioning for autologous peripheral blood stem cell (PBSC) transplantation. Five minutes after starting the infusion when a total of about $10 \mathrm{mg}$ of carboplatin had been administered, she suddenly developed tachypnea, chest tightness, cyanosis, vomiting, cough, hemoptysis, and hypotension. The infusion was immediately interrupted and she was resuscitated with oxygenation, fluid therapy, steroids, and antihistaminic. The chest x-ray, showing bilateral, widespread pulmonary infiltrates along with hemoptysis, suggested diffuse pulmonary hemorrhage, echocardiogram showed reduced cardiac contractility with wall dyskinesia, she was further treated with 1carnitine. Five days after the episodes, follow-up echocardiography disclosed normalization of cardiac contractility. One week later, she was readmitted to undergo PBSC transplantation. Skin test to carboplatin was negative. The same conditioning regimen was used. Carboplatin was administered successfully after premedication with an oral dose of diphenhydramine $50 \mathrm{mg}$ and 3 doses of prednisolone $25 \mathrm{mg}, 13,7$ and 1 hour beforehand (13). Shlebak et al (23) in 1995 reported a 49-year-old woman with relapsing ovarian cancer who developed a HSR to carboplatin and, subsequently, to cisplatin. This patient was known to be allergic to co-amoxiclav and talc, both giving rise to a transient macular skin rash, but had no other history of atopy. They concluded that hypersensitivity to carboplatin, switching to cisplatin-containing cytostatic regimens was tolerated well in most, but not in all patients. Windom et al (24) in 1992 reported a case of successful retreatment with carboplatin after desensitization in a patient with ovarian adenocarcinoma. Abe et al (25) in 2010 reported 3 cases of HSRs to platinum-based chemotherapy drugs that required platinum readministration. Two patients (case 1 and 2) were treated with the desensitization protocol successfully without developing heart rate (HR) during the 
subsequent 3 courses. These cases show the usefulness and effectiveness of the desensitization protocol for the continuation of platinum treatment in patients who have undergone an extended number of treatments.

\section{Conclusion}

HSRs to cisplatin and carboplatin can be a potentially life-threatening complication. These reactions have been increased due to the growing use of these agents in chemotherapy. The symptoms can range from a mild rash to severe anaphylaxis. Doctors should be aware of these reactions, determine appropriate treatment, and know the cross-reactivity among these drugs. Desensitization and skin testing can be helpful to avoid life-threatening accidents.

\section{Acknowledgements}

We express our sincere thanks to the staff of the emergency and neurosurgery department of Imam Khomeini Hospital who participated in this study.

Ethical issues

Confidentiality of patient information was maintained.

\section{Authors' contributions}

All authors passed four criteria for authorship contribution based on recommendations of the International Committee of Medical Journal Editors(ICJME).

\section{Financial Disclosure}

There was no financial support.

\section{References}

1. Jamieson ER, Lippard SJ. Structure, recognition, and processing of cisplatin-DNA adducts. Chem Rev 1999; 99(9): 2467-98.

2. Charalabopoulos K, Karkabounas S, Ioachim E, Papalimneou V, Syrigos K, Evangelou A, et al. Antitumour and toxic effects on Wistar rats of two new platinum complexes. Eur J Clin Invest 2002; 32(2): 129-33.

3. Covens A, Carey M, Bryson P, Verma S, Fung Kee Fung M, Johnston M. Systematic review of first-line chemotherapy for newly diagnosed postoperative patients with stage II, III, or IV epithelial ovarian cancer. Gynecol Oncol 2002; 85(1): 71-80.

4. Ozols RF, Bundy BN, Greer BE, Fowler JM, ClarkePearson D, Burger RA, et al. Phase III trial of carboplatin and paclitaxel compared with cisplatin and paclitaxel in patients with optimally resected stage III ovarian cancer: a Gynecologic Oncology Group study. J Clin Oncol 2003; 21(17): 3194-200.

5. Syrigos KN, Vansteenkiste J, Parikh P, von Pawel J, Manegold C, Martins RG, et al. Prognostic and predictive factors in a randomized phase III trial comparing cisplatin-pemetrexed versus cisplatin- gemcitabine in advanced non-small-cell lung cancer. Ann Oncol 2010; 21(3): 556-61.

6. Joy J, Nair CK. Amelioration of Cisplatin induced nephrotoxicity in Swiss albino mice by Rubiacordifoliaextract. J Cancer Res Ther 2008; 4(3): 111-5.

7. Kannarkat G, Lasher EE, Schiff D. Neurologic complications of chemotherapy agents. Curr Opin Neurol 2007; 20(6): 719-25.

8. Maina A, Richiardi G, Danese S, Defabiani E, Giardina G. Symptomatic hypocalcemia and hypomagnesiemia in cisplatinum-based chemotherapy treated patients: case report. Eur J Gynaecol Oncol 1996; 17(4): 281-2.

9. Callahan MB, Lachance JA, Stone RL, Kelsey J, Rice LW, Jazaeri AA. Use of cisplatin without desensitization after carboplatin hypersensitivity reaction in epithelial ovarian and primary peritoneal cancer. Am J Obstet Gynecol 2007; 197(2): 199.e1-4.

10. Mohammadianpanah M, Omidvari S, Mosalaei A, Ahmadloo N. Cisplatin-induced hypokalemic paralysis. Clin Ther 2004; 26(8): 1320-3.

11. Koren C, Yerushalmi R, Katz A, Malik H, Sulkes A, Fenig E. Hypersensitivity reaction to cisplatin during chemoradiation therapy for gynecologic malignancy. Am J Clin Oncol 2002; 25(6): 625-6.

12. Markman M, Kennedy A, Webster K, Elson P, Peterson G, Kulp B, et al. Clinical features of hypersensitivity reactions to carboplatin. J Clin Oncol 1999; 17(4): 1141.

13. Kook H, Kim KM, Choi SH, Choi BS, Kim HJ, Chung SY, et al. Life-threatening carboplatin hypersensitivity during conditioning for autologous PBSC transplantation: successful rechallenge after desensitization. Bone Marrow Transplant 1998; 21(7): 727-9.

14. Sliesoraitis S, Chikhale PJ. Carboplatin hypersensitivity. Int J Gynecol Cancer 2005; 15(1): 13-18.

15. Morris DJ, Carr B. Hypersensitivity reaction to carboplatin followed by reaction to cisplatin. Community Oncol. 2005; 324-326. Available from: http://www.oncologypractice.com/co/journal/ articles/0204324b.pdf

16. Novak KM. Drug Facts and Comparisons. 59th ed. Chicago, Ill: Wolters Kluwer Health; 2005.

17. Weidmann B, Muelleneisen N, Bojko P, Niederle N. Hypersensitivity reactions to carboplatin. Report of two patients, review of the literature, and discussion of diagnostic procedures and management. Cancer 1994; 73(8): 2218-22.

18. Tonkin KS, Rubin P, Levin L. Carboplatin hypersensitivity: case reports and review of the literature. Eur J Cancer 1993; 29(9): 1356-7.

19. Ottaiano A, Tambaro R, Greggi S, Prato R, Di Maio M, Esposito G, et al. Safety of cisplatin after severe hypersensitivity reactions to carboplatin in patients 
with recurrent ovarian carcinoma. Anticancer Res 2003; 23(4): 3465-8.

20. Libra M, Sorio R, Buonadonna A, Berretta M, Stefanovski P, Toffoli G, et al. Cisplatin may be a valid alternative ap-proach in ovarian carcinoma with carboplatin hypersensitivity: report of three cases. Tumori 2003; 89(3): 311-3.

21. Shukunami K, Kurokawa T, Kubo M, Kaneshima M, Kamitani N, Kotsuji F. Hypersensitivity reaction to carboplatin during treatment for ovarian cancer: successful resolution by replacement with cisplatin. Tumori 1999; 85(4): 297-8.

22. Jones R, Ryan M, Friedlander M. Carboplatin hypersensitivity reactions: re-treatment with cisplatin desensitisation. Gynecol Oncol 2003; 89(1): 112-5.

23. Shlebak AA, Clark PI, Green JA. Hypersensitivity and cross reactivity to Cisplatin and analogues. Cancer Chemother Pharmacol 1995; 35(4): 349-51.

24. Windom $\mathrm{HH}, \mathrm{McGuire} \mathrm{WP}$ 3rd, Hamilton RG, Adkinson NF Jr. Anaphylaxis to carboplatin, a new platinum chemotherapeutic agent. J Allergy Clin Immunol 1992; 90(4 Pt 1): 681-3.

25. Abe A, Ikawa H, Ikawa S. Desensitization treatment with cisplatin after carboplatin hypersensitivity reaction in gynecologic cancer. J Med Invest 2010; 57(1-2): 163-7. 